\title{
Snake Energy Analysis and Result Validation for a Mobile Laser Scanning Data-Based Automated Road Edge Extraction Algorithm
}

\author{
Pankaj Kumar, Paul Lewis, Conor P. McElhinney, Pawel Boguslawski, and Tim McCarthy
}

\begin{abstract}
The negative impact of road accidents cannot be ignored in terms of the very sizeable social and economic loss. Road infrastructure has been identified as one of the main causes of the road accidents. They are required to be recorded, located, measured, and classified in order to schedule maintenance and identify the possible risk elements of the road. Toward this, an accurate knowledge of the road edges increases the reliability and precision of extracting other road features. We have developed an automated algorithm for extracting road edges from mobile laser scanning (MLS) data based on the parametric active contour or snake model. The algorithm involves several internal and external energy parameters that need to be analyzed in order to find their optimal values. In this paper, we present a detailed analysis of the snake energy parameters involved in our road edge extraction algorithm. Their optimal values enable us to automate the process of extracting edges from MLS data for tested road sections. We present a modified external energy in our algorithm and demonstrate its utility for extracting road edges from low and nonuniform point density datasets. A novel validation approach is presented, which provides a qualitative assessment of the extracted road edges based on direct comparisons with reference road edges. This approach provides an alternative to traditional road edge validation methodologies that are based on creating buffer zones around reference road edges and then computing quality measure values for the extracted edges. We tested our road edge extraction algorithm on datasets that were acquired using multiple MLS systems along various complex road sections. The successful extraction of road edges from these datasets validates the robustness of our algorithm for use in complex route corridor environments.
\end{abstract}

Index Terms-Complex road, mobile laser scanning (MLS), road edges, snake energy, validation approach.

Manuscript received January 18, 2016; revised April 6, 2016; accepted April 21, 2016. Date of publication May 18, 2016; date of current version January 23, 2017. Research presented in this paper was initially funded by the Irish Research Council Enterprise Partnership Scheme and the Science Foundation Ireland Strategic Research Cluster grant (07/SRC/I1168). Continued funding included SFI Industrial Fellowship (13/IF/I2782) and contributions from the publication funds of both Maynooth University and the National Centre for Geocomputation. The research was continued at Universiti Teknologi Malaysia. The authors gratefully acknowledge this support. The authors also acknowledge the 3D Laser Scanning Company for providing the Streetmapper mobile laser scanning data. (Corresponding author: Pankaj Kumar.)

P. Kumar is with the Department of Geoinformation, Universiti Teknologi Malaysia, Johor Bahru 81310, Malaysia (e-mail: pankajkumar@utm.my).

P. Lewis, C. P. McElhinney, and T. McCarthy are with the National Centre for Geocomputation, Maynooth University, Maynooth, Ireland (e-mail: paul.lewis@ nuim.ie; conormce@cs.nuim.ie; tim.mccarthy@nuim.ie).

P. Boguslawski is with the Department of Architecture and the Built Environment, University of the West of England, Bristol BS16 1QY, U.K. (e-mail: pawel.boguslawski@uwe.ac.uk).

\section{INTRODUCTION}

$\mathbf{R}$ OAD accidents have become one of the main concerns for policy makers and road infrastructure developers due to thousands of deaths and the economic loss caused by them. Each year, around 1.25 million people die in road crashes around the world, while another 20-50 million are severely injured [1]. Furthermore, these accidents cost between $1 \%$ and $2 \%$ of a country's annual gross national product. According to the World Health Organisation report, road traffic accidents are likely to become the seventh leading cause of death in the world by 2030 [1]. The main challenge for policy makers is to ensure that road networks are as safe as possible while maintaining quality and mobility.

Recent research investigations have described a significant correlation between road infrastructure and accident analysis values [2]. Road design has an immediate effect on accident risk as it influences driver behavior in terms of speed, acceleration, and lateral position. Road user safety may be affected by road geometry and physical features along the route corridor. These factors are required to be recorded, located, measured, and classified in a timely cost-effective manner in order to schedule maintenance and ensure maximum safety conditions for road users. Safe roadway infrastructure has an important role in reducing the accident risk as it contributes to one out of three fatal accidents [3]. Road safety considerations must result in a road environment that should be self-explaining and forgiving, in the sense that users are not faced with unexpected situations and their mistakes can be, if not avoided, corrected [4].

Accurate information about the road and its features is essential for effective management of road networks and to ensure maximum safe driving conditions for road users. This information can assist decision makers to identify the possible risk elements of the road, which may present a safety concern for the driving conditions. With the potential of Geographic Information Science technologies in road management, mobile laser scanning (MLS) systems present a rapid, reliable, and cost-effective tool for carrying out inspections along the route corridor. The European Road Safety Inspection research project demonstrated that mobile mapping systems could be used to collect physical route corridor information for rapid safety analysis [5].

MLS systems can be employed to capture 3-D spatially referenced information about the road and its surrounding environment. The use of light detection and ranging (LiDAR) technology for mapping route corridors enables accurate acquisition 
of dense 3-D point cloud data which contain elevation, intensity, pulsewidth, range, and multiple echo attributes. These data attributes can be used for reliable and precise extraction of different road features. The road edge is a fundamental feature and its correct identification is a prerequisite in order to obtain precise information about road geometry and physical road objects. We have developed an automated algorithm for extracting road edges from MLS data [6]. This algorithm is based on a novel combination of two modified versions of the parametric active contour or snake model. In the parametric model, the snake is represented explicitly as a controlled spline curve, which is implemented based on computed energy [7]. It is defined within a 2-D image domain that moves toward a desired object boundary under the influence of an internal energy within the curve itself and an external energy derived from the image data. The internal energy is applied to the snake curve which controls the curve's elasticity and rigidity, while the external energy attracts the snake curve toward the object boundary. The movement of the snake curve is controlled through balancing the internal and external energy terms until an energy minimization condition is met. When the snake's energy function reaches a minimum, it converges to the object boundary [8]. We used the parametric active contour model in our algorithm, since its implementation is less computationally expensive when compared to geometric active contour models. In the geometric model, the curve is represented implicitly as a level set and is evolved based on geometric computations, with its speed locally dependent on the image data [9], [10].

The internal and external energy parameters involved in our algorithm have been analyzed in order to find their optimal values, which enables the snake curve to precisely converge to the road edges. In this paper, we present a detailed investigation of these parameters based on assessing the internal and external energy properties of the snake curve. We have investigated how low and nonuniform point density affects the performance of extracting features from LiDAR dataset. This was achieved by adding a modified balloon external energy in our algorithm, and we demonstrate how its modified version can be useful in cases where datasets have a low and nonuniform point density along the road section. The majority of the road edge validation approaches are based on computing quality measure values with respect to the buffer zones created around reference road edges. We present a more efficient and automated approach for validating the extracted road edges based on their direct comparison with reference road edges.

In Section II, we review various methods, which have been developed for extracting roads and its boundaries from LiDAR data. We present a detailed review of the validation approaches, which have been used to verify the extracted road boundaries. Following the review, we list the limitations in current road extraction and their validation approaches, which have been addressed through our research work. In Section III, we provide a brief description of our road edge extraction algorithm. In Section IV, we present a detailed experimental analysis of the internal and external energy parameters involved in our algorithm. In Section V, we present the modified balloon external energy component in our algorithm. In Section VI, we present an automated approach for validating the road edges extracted from MLS data. In Section VII, we test our algorithm on the datasets acquired using multiple MLS systems along a variety of road sections. These test road sections consisted of distinct sets of edges, roundabout, and high degree of curvature datasets. In Section VIII, we validate the extracted road edges and discuss the test results. Finally, we conclude our paper in Section IX.

\section{LITERATURE REVIEW}

The MLS system provides 3-D point cloud data, which can be useful for extracting road features. Most methods developed for extracting road features are based on identification of planar or smooth surfaces and the classification of point cloud data on the basis of its attributes [11]. A robust evaluation of the extracted road features is also important for assessing the relevance of method for practical applications [12].

Elberink and Vosselman [13] reconstructed 3-D road models by assigning airborne LiDAR points to 2-D topographic map polygons. The height values of map points were calculated by fitting a least-squares plane to the LiDAR points inside the polygon. The precision of map point heights was calculated from error propagation based on laser point noise, systematic laser data errors, and plane fitting uncertainty components. The quality of a reconstructed 3-D model was estimated against the photogrammetric derived topographic database [14]. Jaakkola, Hyyppä, Hyyppä, and Kukko [15] delineated road kerb by filtering the gradient image of elevation information obtained from the MLS system. Their evaluation approach was based on calculating completeness, correctness, and mean accuracy values against manual classification of the dataset. Goepfert and Rottensteiner [16] applied the snake model to extract road network from airborne LiDAR data. In their approach, the snake curve was initialized near the road feature using available vector data, while the external energy terms were derived from elevation, intensity and surface roughness information obtained from the LiDAR points. The orthophotos were manually digitized to create reliable reference data for validation purpose. The extracted road network was validated by finding a mean point-to-contour distance between the reference and the final position of the snake curve. Boyko and Funkhouser [17] presented a method for extracting urban roads from a large-scale mobile and aerial laser scanning datasets. An initial approximation of the road network in the point cloud was made using 2-D map, while the elevationbased attractor function was used as an external energy of the snake to find the road edges. The road detection results were evaluated by comparing them with a manually created $2-\mathrm{D}$ road grid. The LiDAR points predicted as road were projected into the 2 -D grid, and then, the value of each grid cell was set to 1 if it contains the point, otherwise 0 . These values were used to compute the accuracy metrics such as correctness, completeness, quality, average spill size, and direction, where spill represents the distance of the extracted road side from the manually extracted road side. Ibrahim and Lichti [18] extracted street kerb and its surface by applying the derivate of the Gaussian function to the ground segment refined from MLS data. The extracted kerbs were evaluated by measuring its distance from manually extracted kerb at each point. The K-D (K-dimensional) tree approach was used to find the nearest point in the manually extracted kerb dataset 
with respect to each point in the extracted kerb dataset. Due to high point density, the measured distance was assumed as close to the normal distance between the two kerbs.

Zhou and Vosselman [19] presented a three-step approach for mapping kerbs from mobile and airborne laser scanning data. First, small-height jumps were detected near the terrain surface; then, a sigmoidal function was fitted to mid points of the height jump, and finally, small gaps between nearby and collinear line segments were closed. They analyzed the extraction results by computing completeness and correctness measure values with reference to the buffer zone created around manually outlined road sides in orthophotos. The geometrical accuracy of the extracted kerbs was also evaluated by making their comparison with global positioning system (GPS) measurements. Serna and Marcotegui [20] presented an approach for automated analysis of urban accessibility using MLS data. In their approach, interpolated 2.5-D range image was used to segment building facades, urban objects, and road kerbs. The evaluation of kerb extraction results was based on computing the completeness and correctness measures against the buffer zone created around manually extracted kerb lines. Yang, Fang, and Li [21] developed a method for extracting and delineating road kerbs from large-scale MLS data. The point clouds were partitioned into road cross sections on the basis of GPS time associated with points, and then, a moving window operator was used to filter out nonground points. The kerbs were located by analyzing elevation jump, point density, and slope change for a set of points, and finally, the extracted kerbs were refined to eliminate the false segments. The extraction results were validated by computing the quality measure values with respect to the manually digitized road kerbs. Guan et al. [22] presented a stepwise approach to extract road markings from MLS point cloud. In their approach, vehicle trajectory data were used to partition raw MLS data, and then, kerb edges were extracted by detecting height jumps using slope and elevation-difference thresholds. Next, the estimated road surface points were interpolated into intensity image using inverse distance weighted approach, and then, point-density-dependent multiple thresholds were applied to segment road marking candidates. Finally, morphological operations were applied to refine the road markings by removing noise and improving completeness. The road marking outputs were evaluated by making their comparison with manual interpretation, while the validation of kerb edges was reported in [23] based on estimating their Euclidean distance with reference ground points collected using total station. Wang et al. [24] developed a method for detecting kerb edges from MLS point cloud in an urban environment. The point cloud data were partitioned into a number of overlapping data blocks based on vehicle trajectory, and then, salient points in each block were extracted by projecting the distance of each point's normal vector to the point cloud's dominant vector into a hyperbolic tangent function space. The kerb edges were extracted from the salient points based on their elevation, horizontal length, and distance to the trajectory. Finally, the kerb points of adjacent data blocks were merged and fitted into smooth curves through a curve fitting algorithm. The extracted road boundaries were validated against manual extraction by computing completeness, correctness, and quality values.
Recent years have seen some progress toward the development of automated approaches for extracting road features from LiDAR datasets. However, most of the approaches have been developed for extracting kerb edges in urban road environments where the algorithms rely on the existence of a sufficient height or slope difference between the road and kerb points. Little research has been focused on extracting edges in rural and semiurban areas where the road edges distinguish the road surface from grass-soil [6]. LiDAR intensity and pulse width attributes can be a useful source of information for extracting edges, which are needed to be thoroughly explored. The values of input parameters are critical for the performance of any automated feature extraction method. The input parameters are required to be analyzed experimentally in order to automate the process of extracting road edges.

An efficient and robust validation of the road extraction results have been little focused by the researchers. Most of the road validation approaches are based on creating the buffer zone around the reference data, and then, the extracted road is matched within the buffer to manually compute quality measure values. The computed quality measure values provide an aggregate evaluation of the extracted road edges rather than evaluating individual road edge points [6]. An appropriate selection of buffer width is another issue, which is required to be considered. If the buffer width is large, false extractions will be incorrectly considered as road, but if its value is small, correct extractions will be rejected. The applied validation approaches are also accompanied with a manual computation of quality measure values. Some approaches are based on evaluating the results by finding nearest or mean distance between the reference and the extracted road points rather than finding orthogonal distance. There is a need to develop more robust and automated approach for qualitative validation of the road extraction results. In the next section, we provide a brief overview of our road edge extraction algorithm.

\section{ROAd EDGE EXTRACTION AlgORITHM}

Our algorithm extracts road edges from MLS data using the novel combination of a gradient vector flow (GVF) and balloon parametric active contour or snake models. A workflow of the road edge extraction algorithm is shown in Fig. 1. In our algorithm, the MLS data are preprocessed to input $n$ number of $30 \mathrm{~m}$ $\times 10 \mathrm{~m} \times 5 \mathrm{~m}$ LiDAR point clouds and $n$ number of $30-\mathrm{m}$ navigation data sections. This preprocessing is done automatically based on positional and heading information of the MLS survey vehicle along the selected road section. The dimensions of input data sections are selected based on experimental analysis [25]. The input data sections are selected with an overlap of 2 $\mathrm{m}$ between them, which allows us to batch process consecutive and overlapped road sections as required in the algorithm. We use the LiDAR elevation, reflectance, and pulse width attributes in the algorithm, which are converted into 2.5-D raster surfaces in order to reduce computational expense. In Step 1 of our algorithm, multiresolution terrain pyraminds are generated from the LiDAR attributes. In Step 2, 2.5-D raster surfaces are estimated from the first level terrain pyramids using natural neighborhood interpolation [26], [27]. The cell size $c$ parameter, required to 


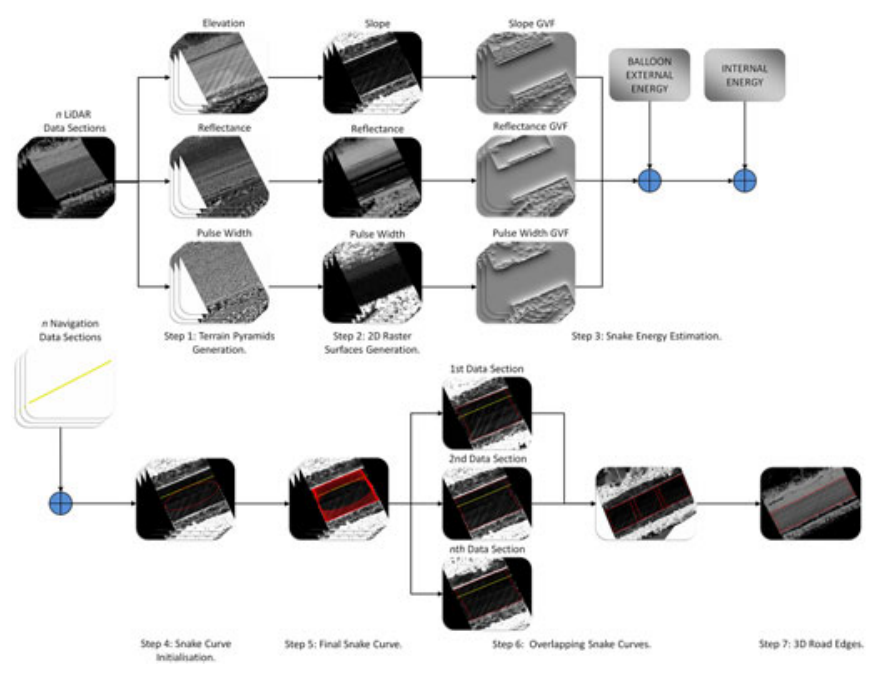

Fig. 1. Road edge extraction algorithm components [6].

generate the raster surfaces, is selected based on an experimental analysis. This analysis was done as the value of cell size may affect the accuracy and computational cost of our algorithm. To find its optimal value, we analyzed the temporal, completeness, and accuracy performance of our road edge extraction algorithm in raster surfaces generated with different cell sizes [25]. Slope values are estimated from the elevation raster surface as the rate of change in elevation of the raster cells to its neighbors. The slope, reflectance, and pulse width raster values are normalized with respect to their global minimum and maximum values, and converted to an 8-bit data type.

In Step 3 of our road edge extraction algorithm, internal and external energy terms are estimated. The internal energy is provided to the snake curve by adjusting its elasticity and stiffness properties with $\alpha$ and $\beta$ weight parameters, while the step size of the snake curve is controlled with a $\gamma$ weight parameter. The values of these parameters are estimated based on experimental analysis as presented in Section IV-A. The GVF external energy terms are computed as a diffused energy field of the gradient vectors of the object boundaries from the raster surfaces [28]. In order to compute the GVF energy, we estimate the object boundaries from the slope, reflectance, and pulse width raster surfaces through the consecutive use of hierarchical thresholding and Canny edge detection. In the hierarchical thresholding approach, a hierarchy of low-to-high-resolution versions of input raster surfaces is created using a mask size, $M$. Then, a threshold $T$ is applied at each hierarchical level which leads to a precise estimation of objects [29]. The mask size $M_{\text {slope }}, M_{\text {ref }}$, $M_{\mathrm{pw}}$ and threshold $T_{\mathrm{slope}}, T_{\mathrm{ref}}, T_{\mathrm{pw}}$ parameters applied to the slope, reflectance, and pulse width raster surfaces are found empirically. Their values are fixed for all the road sections, which allow for the fully automatic application of hierarchical thresholding. The hierarchical thresholding approach outputs raster surfaces with only two values, 255 for object cells and 0 for nonobject cells. We input these surfaces into the Canny edge detection in which the upper threshold $T_{1}$ and the lower threshold $T_{2}$ parameters are applied, which provides an estimation of the object boundaries [30]. The values of the $T_{1}$ and $T_{2}$ parameters are set as 250 and 5, respectively; this is based on the output cell values obtained from the hierarchical thresholding, which makes our Canny edge detection fully automated. The balloon external energy is included by providing a weight to the normal unit vector of the snake points with the $\kappa_{1}$ parameter. Thus, GVF external energy terms attract the snake curve, while balloon external energy helps push it outwards toward the object boundaries. The weight of the slope, reflectance, and pulsewidth-based GVF energy terms are set with the $\kappa_{2}, \kappa_{3}$, and $\kappa_{4}$ parameters, respectively. The values of the weight parameters for the GVF and balloon energy terms are estimated based on experimental analysis, as presented in Section IV-B.

In Step 4, the snake curve is initialized over a 2.5-D raster surface based on the MLS survey vehicles navigation track along each road section. We initialize the snake curve in the form of a parametric ellipse using $\phi$ and $\omega$ angle parameters. The $\phi$ angle is calculated from the average heading angle of the survey vehicle along each road section under investigation, while the $\omega$ angle is selected empirically and fixed for the road sections with similar width. The number of points in the snake curve are determined using the $\delta \lambda$ parametric angle interval, which varies from 0 to $2 \pi$ rad, while the impact of any noise present in the raster surface is controlled with a $\mu$ regularization parameter. The values of the $\delta \lambda$ and $\mu$ parameters are estimated empirically and fixed for all the road sections. In Step 5, the snake curve moves under the influence of internal and external energy terms. It approaches the minimum energy state and converges to the road edges during an iterative process. In Step 6, we obtain overlapping snake curves by batch processing consecutive individual road sections. The intersection points in between the overlapping snake curve points are found, and then, nonroad edge points in between the intersection points are removed. This results in one continuous snake with the left and right edges for the complete road section defined. Finally, in Step 7, the third dimension is defined for the road edge points by finding the elevation value from the nearest LiDAR point to the road edge point. In the next section, we provide a detailed analysis of the internal and external energy parameters.

\section{SNAKE ENERGY ANALYSIS}

In this section, the internal and external energy parameters are experimentally analyzed to estimate their optimal values. The use of optimal values in our algorithm automates the process of extracting road edges from MLS data for all road sections. To perform this analysis, we selected one 10-m section of the N4 national primary road, in County Westmeath, Ireland, which consisted of grass-soil edges and embankments along both sides. We used one $30 \mathrm{~m} \times 10 \mathrm{~m} \times 5 \mathrm{~m}$ section of LiDAR data and one $10-\mathrm{m}$ section of navigation data to process the selected road section. The processed data were collected using the eXperimental Platform (XP-1) MLS system, which has been designed and developed at Maynooth University (MU) [8]. The LiDAR data included elevation, reflectance, and pulse width attributes, which were used in the algorithm to move the snake curve towards road edges. Several test cases considered the internal and 
TABLE I

PARAMETERS USED IN EACH TEST CASE OF INTERNAL AND EXTERNAL ENERGY ANALYSIS

\begin{tabular}{lccccc}
\hline \hline Parameter & Value & Parameter & Value & Parameter & Value \\
\hline$c$ & $0.06 \mathrm{~m}^{2}$ & $T_{\text {slope }}$ & 45 & $T_{2}$ & 5 \\
$M_{\text {slope }}$ & 5 & $T_{\text {ref }}$ & 95 & $\omega$ & $34^{\circ}$ \\
$M_{\text {ref }}$ & 5 & $T_{\mathrm{p} \mathrm{w}}$ & 10 & $\delta \lambda$ & 0.03 \\
$M_{\text {pw }}$ & 5 & $T_{1}$ & 250 & $\mu$ & 0.2 \\
\hline \hline
\end{tabular}

TABLE II

VALUES OF $\alpha, \beta$, AND $\gamma$ WeIght PARAMETERS Used IN THE SiX TEST CASES OF INTERNAL ENERGY ANALYSIS

\begin{tabular}{lccc}
\hline \hline Test Case & $\alpha$ & $\beta$ & $\gamma$ \\
\hline 1 & 0 & 0 & 1 \\
2 & 9 & 0 & 1 \\
3 & 9 & 9 & 1 \\
4 & 9 & 0.001 & 1 \\
5 & 9 & 0.001 & 3 \\
6 & 9 & 0.001 & 9 \\
\hline \hline
\end{tabular}

external energy by using different weight parameters to analyze the performance of our algorithm. In each test case, the parameters used in our algorithm are shown in Table I. The value of $\phi$ was calculated from the average heading angle $\theta$ of the survey vehicle moving along the road section. The number of GVF iterations was 600 , while the number of iterations required to move the snake curve was 40 . In the following sections, we present different test cases, which were considered for analyzing the internal and external energy.

\section{A. Internal Energy}

We considered six test cases in which the performance of our algorithm was analyzed with different values of $\alpha, \beta$, and $\gamma$ internal energy parameters, as shown in Table II. The values of external energy parameters were selected as $\kappa_{1}=2, \kappa_{2}=2$, $\kappa_{3}=2$, and $\kappa_{4}=2$ in each test case. A proper analysis of the internal energy parameters requires that the movement of snake curve is to the left and right edges. Keeping this in mind, we empirically selected those values of external energy parameters which would enable the snake to reach the road edges. In each test case, we applied our road edge extraction algorithm to the road section. The final positions of the snake curve in the six test cases are shown in Fig. 2.

In the first case, we started with the lowest possible values for the $\alpha, \beta$, and $\gamma$ parameters. This caused the snake curve to be jagged at most of the points, as shown in Fig. 2(a). In the second case, the value of $\alpha$ was increased to 9 along with $\beta=0$ and $\gamma=1$, which caused the snake curve to move toward the road edges without any jaggedness, as shown in Fig. 2(b). The $\alpha$ parameter is used to hold the snake curve together and control its elasticity. Its higher value increased the binding energy in the snake curve, while its lower value caused the snake curve to move without any binding energy. Based on this analysis,

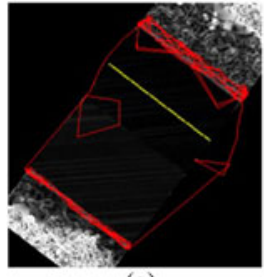

(a)

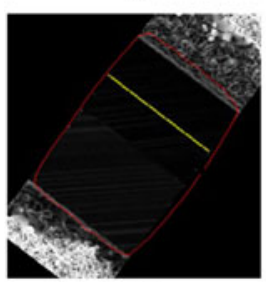

(d)

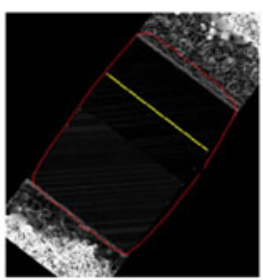

(b)

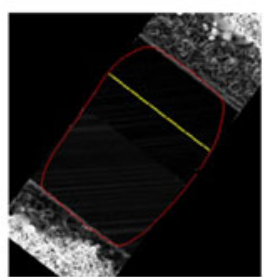

(e)

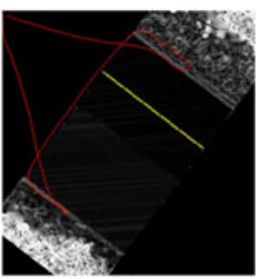

(c)

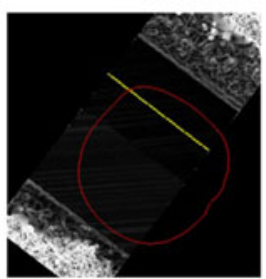

(f)
Fig. 2. Final position of the snake curve is represented in red over the slope raster surface in the (a) first, (b) second, (c) third, (d) fourth, (e) fifth, (f) sixth, and $(\mathrm{g})$ seventh test case of internal energy analysis, while the navigation points are represented in yellow.

we selected an optimal value of 9 for the $\alpha$ parameter when extracting the road edges.

In the third case, the value of $\beta$ was increased to 9 along with an optimal value of $\alpha$ and $\gamma=1$. The effect on the snake curve was extreme binding at some of its points, as can be seen in Fig. 2(c). In the fourth case, we set the minimum value of the $\beta$ parameter to 0.001 along with an optimal value of $\alpha$ and $\gamma=1$. This caused the snake curve to move toward the road edges similar to the movement in the second test case, as shown in Fig. 2(d). The $\beta$ parameter is used to prevent the snake curve from bending and control its stiffness. The lower value allowed the snake curve to move without any bending but its higher value caused extreme bending in the snake curve. The extraction of the road edge does not require much bending in the snake curve so a low value of 0.001 was found to be optimal for the $\beta$ parameter.

In the fifth case, we increased the value of $\gamma$ parameter to 3 along with optimal values of $\alpha$ and $\beta$, which enabled the snake curve to accurately converge to the road edges, as can be seen in Fig. 2(e). In the sixth case, the $\gamma$ parameter value was further increased to 9 along with optimal values for the other internal energy terms. These changes obstructed the movement of the snake curve toward the road edges, as shown in Fig. 2(f). The $\gamma$ parameter controls the step size of the snake curve in one iteration. The higher value led to the controlled step size of the snake curve in one iteration obstructing its growth, while a lower value led to the relatively less controlled step size of the snake curve in one iteration causing its free movement beyond the left edge as in the second and fourth cases. Thus, an optimal value of $\gamma$ parameter was selected as 3 for extracting the road edges. This internal energy analysis helped us to understand that the lower values of $\beta$ and $\gamma$ parameters are required, while a relatively higher value of the $\alpha$ parameter is required to force the snake curve to converge more precisely to the road edges. These findings enabled us to select the optimal values of the internal energy parameters in a more efficient and simplified way. 
TABLE III

VALUES OF $\kappa_{1}, \kappa_{2}, \kappa_{3}$, AND $\kappa_{4}$ WEIGHT PARAMETERS USED IN THE SiX TEST CASES OF EXTERNAL ENERGY ANALYSIS

\begin{tabular}{lllll}
\hline \hline Test Case & $\kappa_{1}$ & $\kappa_{2}$ & $\kappa_{3}$ & $\kappa_{4}$ \\
\hline 1 & 0 & 4 & 0 & 0 \\
2 & 0 & 4 & 2 & 0 \\
3 & 0 & 4 & 2 & 2 \\
4 & 0 & 4 & 4 & 4 \\
5 & 1 & 4 & 2 & 2 \\
6 & 4 & 4 & 2 & 2 \\
\hline \hline
\end{tabular}

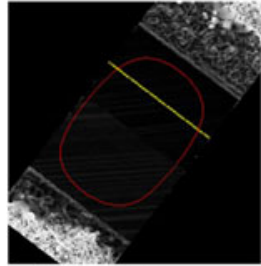

(a)

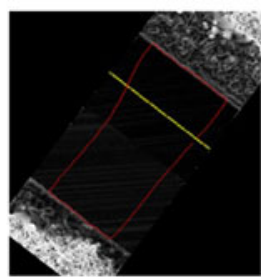

(d)

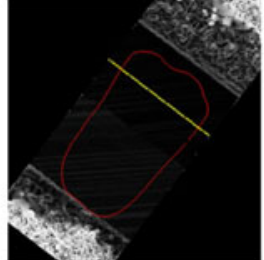

(b)

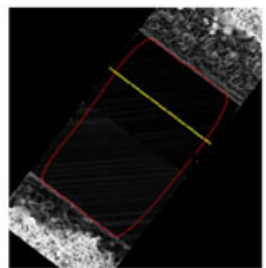

(e)

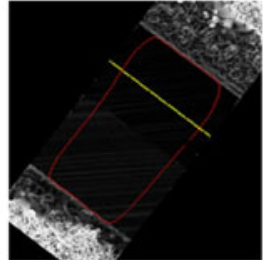

(c)

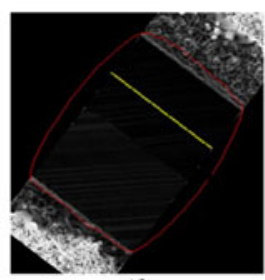

(f)
Fig. 3. Final position of the snake curve is represented in red over the slope raster surface in the (a) first, (b) second, (c) third, (d) fourth, (e) fifth, and (f) sixth case of external energy parameter analysis, while the navigation points are represented in yellow.

\section{B. External Energy}

In this section, we considered six test cases in which the performance of our algorithm was analyzed with the use of various combinations of $\kappa_{1}, \kappa_{2}, \kappa_{3}$, and $\kappa_{4}$ external energy parameters, as shown in shown in Table III. The internal energy parameters were selected as $\alpha=9, \beta=0.001$, and $\gamma=3$. We applied our road edge extraction algorithm in each test case. The final positions of the snake curve in the six test cases are shown in Fig. 3. In the first case of the external energy analysis, we initiated the process with a $\kappa_{2}=4$ weight which provided only a slopebased GVF energy. The applied energy caused the snake curve to move toward the road edges; however, it was not sufficiently strong to force the snake curve to reach the edges, as shown in Fig. 3(a). In the second case, we set $\kappa_{3}=2$, the weight to reflectance-based GVF energy, along with $\kappa_{2}=4$. This caused the snake curve to move further toward the road edges compared with its position in the first case, as can be seen in Fig. 3(b). In the third case, the $\kappa_{4}=2$ weight was set for the pulse-widthbased GVF energy along with $\kappa_{2}=4$ and $\kappa_{3}=2$. This caused the snake curve to converge to both the left and right edges, as shown in Fig. 3(c). In the fourth case, we provided equal weights to the slope, reflectance, and pulse-width-based GVF energy terms with $\kappa_{2}=4, \kappa_{3}=4$, and $\kappa_{4}=4$; however, the snake curve was not able to efficiently converge to the road edges, as can be seen in the third test case shown in Fig. 3(d). The reflectance values provided by the laser scanner in the XP1 MLS system are not accurately normalized which leads to different values over the road surface. The hierarchical thresholding applied to the reflectance raster surface failed to remove the road marking cells near the left road edge which obstructed the snake curve preventing it from moving beyond them. Due to its imperfect normalization, we decided to keep the lower weight for the reflectance-based GVF energy than slope GVF energy. Similarly, we did not choose to set the highest weight to the pulse-width-based GVF energy because the orientation of the kerb edges relative to the vehicle is similar to that of the road surface. This together with their similar surface composition results in the pulse width having similar values which leads to lower priority for the pulse width attribute in urban regions. Based on this, we selected optimal values for $\kappa_{2}, \kappa_{3}$, and $\kappa_{4}$ as 4,2 , and 2, respectively, in the third case.

In the fifth case, the balloon energy was included in the external energy with $\kappa_{1}=1$ along with an optimal values for other GVF energy terms. The balloon energy pushed the snake curve outwards causing it to converge to the road edges better than in the third case, as shown in Fig. 3(e). In the sixth case, we increased the value of $\kappa_{1}$ balloon energy weight to 4 along with an optimal values for other GVF energy terms. The snake curve fully converged to the road edges but also moved beyond the left and right edges as shown in Fig. 3(f). Thus, we selected an optimal value for $\kappa_{1}$ as 1 . However, an increased weight to the balloon energy can be useful in cases of noisy data along the road section which provides an additional inflation energy to the snake curve to overcome the noise. This experimental analysis demonstrated the relative importance of GVF and balloon external energy terms in our algorithm and enabled us to efficiently estimate their optimal values. In the next section, we present a modified balloon external energy in our road edge extraction algorithm.

\section{BALLOON ENERGY MODIFICATION}

Low and nonuniform point density along the road surface affects the performance of our road edge extraction algorithm. This is because the low point density creates a significant amount of noisy cells along the raster surface generated from the LiDAR data. The hierarchical thresholding that is applied to the raster surface is not able to remove these noisy cells, thus creating an obstruction for the snake curve moving toward the edges, as shown in Fig. 4(a). For example, our XP-1 MLS employed a single laser scanner during the data acquisition process which led to the collection of LiDAR data with a lower point density along the right side of the road section than along its left side [31]. It resulted in a large number of noisy cells along the right side of the road section in the slope raster surface generated from the LiDAR elevation attribute. This noise can be overcome by providing an increased weight to the balloon energy parameter with $\kappa_{1}=4$. It provides an additional inflation energy to the snake curve to move it toward the right road edge, as shown in Fig. 4(b). However, the increased balloon energy pushes the snake curve beyond the weak left edge points. This limitation 


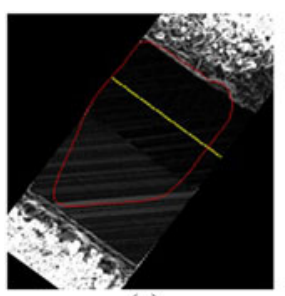

(a)

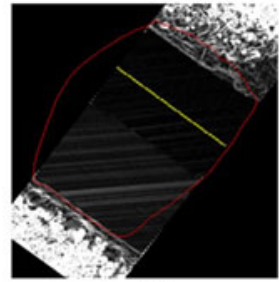

(b)
Fig. 4. Final position of the snake curve is represented in red over the slope raster surface with (a) $\kappa_{1}=1$ and (b) $\kappa_{1}=4$ parameter in $10 \mathrm{~m}$ national primary road section, while the navigation points are represented in yellow.
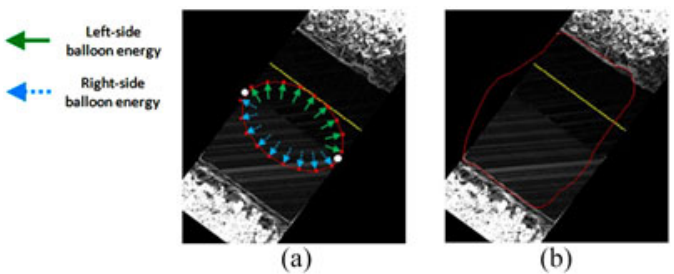

Fig. 5. (a) Initial and (b) final position of the snake curve is represented in red over the slope raster surface with $\kappa_{1}^{\text {left }}=1$ and $\kappa_{1}^{\text {right }}=4$ in a 10-m national primary road section; navigation points are represented in yellow, while end points of major axis of the initial snake curve are represented as white dots.

can also be overcome with a modified balloon energy in our road edge extraction algorithm.

We modified the balloon energy by providing a low balloon energy weight to the snake points toward the left side of the road section while keeping a high weight to the snake points toward the right side of the road section. In this process, end points of major axis of the initial snake curve were first estimated as shown in Fig. 5(a). In each iteration of the algorithm, the snake points nearest to the end points were estimated, and then, the selected snake points were used to divide the snake curve points into the points toward left and right side of the road section. Finally, the balloon energy with $\kappa_{1}^{\text {left }}=1$ was provided to the snake points toward the left side, while the balloon energy with $\kappa_{1}^{\text {right }}=4$ was provided to the snake points toward the right side. During an iterative process, the snake curve moved toward the left and right edges of the road section and converged efficiently to them, as shown in Fig. 5(b). The left-side balloon energy kept the snake from moving beyond the left edge, while the rightside balloon energy provided an additional inflation energy to the snake curve to move toward the right edge. In this way, the use of balloon energy weight parameter was balanced relative to the nonuniform point density of the LiDAR data. In the next section, we present our approach for validating the road edges extracted from MLS data.

\section{ROAD EDGE VALIDATION APPROACH}

This section details an approach for validating the road edges extracted from MLS data. This approach is based on an automated estimation of the road edge's orthogonal proximity from a reference road edge. The reference edge can be based on either manual digitization or a ground truth data collected using field

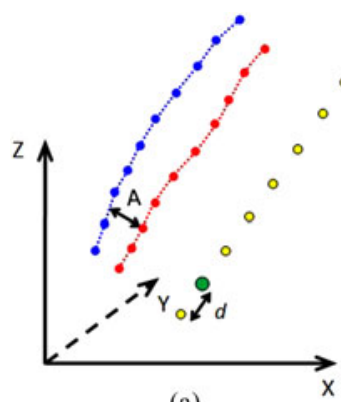

(a)

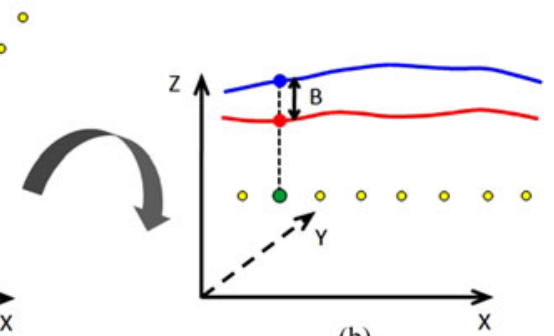

(b)
Fig. 6. Road edge validation approach. (a) Original and (b) rotated positions of extracted and reference road edges, where red represents the extracted road edges, blue represents the reference road edges, yellow represents the navigation points, and green represents the navigation point selected in each iteration.

survey. As shown in Fig. 6(a), an automated estimation of the orthogonal distance $A$ in between the original positions of extracted and reference road edge points would be more complex. This is because the orthogonal position of the reference edge point with respect to any selected extracted edge point is not known. In order to simplify the process of estimating orthogonal distance, the points are rotated toward the horizontal axis based on the navigation data, which provides the heading angle information of the road section with respect to true North.

In our validation approach, the navigation point is selected at some $d$ interval along the road section in each iteration as shown in Fig. 6(a). The extracted and reference road edge points are rotated towards the horizontal $X$-axis based on the heading angle of the selected navigation point. The use of heading information allows us to automate the process of rotating the edge points. After rotation, linear splines are fitted to both the 2-D extracted and reference road edge points as shown in Fig. 6(b). The purpose of fitting a linear spline is to find the 2-D interpolation function, which is then used to interpolate the $Y$-axis values of the extracted and reference edge points with respect to the known $X$-axis value of the selected navigation point. We assign $Z$-axis values to the interpolated 2-D road edge points by assigning the elevation values from the nearest LiDAR points to them. We then calculate an orthogonal Euclidean distance between the extracted and reference 3-D road edge points. The positive or negative sign is assigned to the calculated distance based on the $Y$-axis values of the road edge points. The positive or negative values indicate the inside or outside position of the extracted road edge points with respect to the reference road edge points. This process is iterated for all the navigation points selected at a $d$ interval. Thus, the validation approach provides the orthogonal Euclidean distance values in between the extracted and reference road edge points based on the selected navigation points. In the next section, we present the tests of our road edge extraction algorithm when used on datasets acquired using multiple MLS systems along complex route corridor environments.

\section{EXPERIMENTATION}

We selected three complex sections of road to test our road edge extraction algorithm. These sections covered $245 \mathrm{~m}$ of 


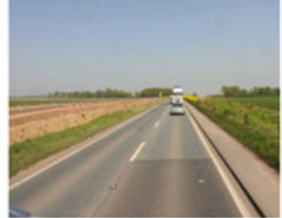

(a)

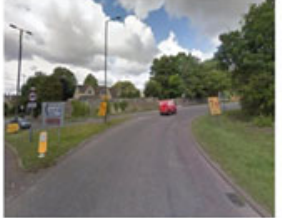

(b)

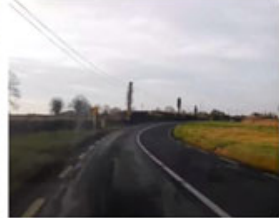

(c)
Fig. 7. Digital image of the (a) first road section consisting of a grass-soil edge along its left side and a kerb edge along its right side in County Nottinghamshire, U.K. (Geographic location: $\left.53^{\circ} 01^{\prime} 00.08^{\prime \prime} \mathrm{N} 0^{\circ} 54^{\prime} 06.3^{\prime \prime} \mathrm{W}\right)(\mathrm{Im}-$ age Courtesy: StreetMapper), (b) second road section consisting of kerb edges along both sides of a roundabout in County Gloucestershire, UK (Geographic location: $51^{\circ} 43^{\prime} 02.2^{\prime \prime} \mathrm{N} 1^{\circ} 57^{\prime} 35.3^{\prime \prime} \mathrm{W}$ ) (Image Courtesy: Streetview, Google), and (c) third road section consisting of grass-soil edges along both sides with a high degree of curvature in County Westmeath, Ireland (Geographic location: $53^{\circ} 33^{\prime} 26.3^{\prime \prime} \mathrm{N} 7^{\circ} 13^{\prime} 29.7^{\prime \prime} \mathrm{W}$ ) (Image Courtesy: Ubipix).

TABLE IV

PARAMETERs USED IN THE AUTOMATEd RoAd EdGE EXTRACTION ALGORITHM

\begin{tabular}{lccccc}
\hline \hline Parameter & Value & Parameter & Value & Parameter & Value \\
\hline$c$ & $0.06 \mathrm{~m}^{2}$ & $T_{\text {int }}$ & 55 & $\kappa_{2}$ & 4 \\
$M_{\text {slope }}$ & 5 & $T_{\mathrm{pw}}$ & 10 & $\kappa_{3}$ & 2 \\
$M_{\text {ref }}$ & 5 & $T_{1}$ & 250 & $\kappa_{4}$ & 2 \\
$M_{\text {int }}$ & 5 & $T_{2}$ & 5 & $\omega$ & $20^{\circ}$ \\
$M_{\text {pw }}$ & 5 & $\alpha$ & 9 & $\delta \lambda$ & 0.03 \\
$T_{\text {slope }}$ & 45 & $\beta$ & 0.001 & $\mu$ & 0.2 \\
$T_{\text {ref }}$ & 95 & $\gamma$ & 3 & & \\
\hline \hline
\end{tabular}

road which consisted of distinct sets of edges, roundabout, and high degree of curvature. The first $50-\mathrm{m}$ section of minor road consisted of a grass-soil edge along its left side and a kerb edge along its right side as shown in Fig. 7(a). The second 105-m section of primary route network consisted of kerb edges along both sides of a roundabout as shown in Fig. 7(b), while the third 90-m section of national primary road consisted of grass-soil edges along both sides with a high degree of curvature, as shown in Fig. 7(c). The lengths of these road sections were selected such that the distinct edges, roundabout and high degree of curvature features could be included in our algorithm tests. The processed datasets for the first, second, and third road sections were acquired using StreetMapper [32], RouteMapper [33], and XP-1 MLS systems, respectively. These datasets, from multiple MLS systems, were selected to validate the robustness of our algorithm. To process 50-, 105-, and 90-m road sections, we used $n=6,13$, and 11 sets of $30 \mathrm{~m} \times 10 \mathrm{~m} \times 5 \mathrm{~m}$ sections of LiDAR data, respectively, while $n=6,13$, and 11 sets of $10-\mathrm{m}$ sections of navigation data were used.

The parameters used in our algorithm are shown in Table IV.

In the first road section, the acquired data contained intensity values which enabled us to use the mask size $M_{\text {int }}$ and threshold $T_{\text {int }}$ parameters in our algorithm. The data did not include a pulse width attribute; thus, our algorithm was applied based on elevation and intensity attributes only. In the second road section, the algorithm was applied based on only elevation and pulse width attributes, while in the third road section, we used elevation, reflectance, and pulse width attributes. The value of $\phi$ was calculated from the average heading angle $\theta$ of the survey

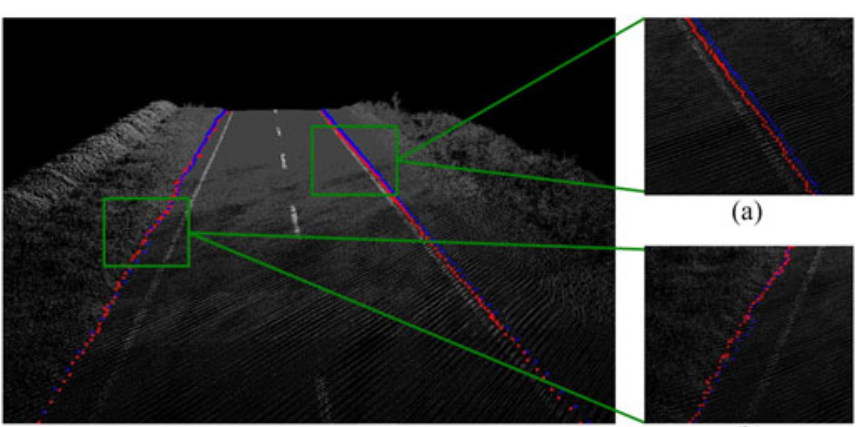

(b)

Fig. 8. Automatically extracted 3-D edges are represented in red and the manually digitized 3-D edges are represented in blue in the first 50-m road section. The inset pictures of (a) left and (b) right edges show some of the points in detail.

vehicle along each road section. The number of GVF iterations was 600 , while the number of iterations required to move the snake curve was 40 .

In the first road section, the data were acquired using a single laser scanner with a double-pass approach in which the vehicle was driven back and forth on the road section. This led to uniform and dense point cloud data along the first road section in comparison with the second and third road sections, where the data were acquired using a single laser scanner and only a single-pass approach. Moreover, we did not utilized the reflectance attribute in the second road section due to which the snake curve was not able to converge fully to the road edges in most of the cases. This caused us to apply a modified balloon energy with $\kappa_{1}^{\text {left }}=1$ and $\kappa_{1}^{\text {right }}=2$ in the second road section. In the first road section, the point density was uniform and dense, while in the third road section, we utilized all the LiDAR attributes, allowing us to define the balloon energy with $\kappa_{1}^{\text {left }}=1$ and $\kappa_{1}^{\text {right }}=1$. We applied our automated road edge extraction to each road section with the optimally selected parameters. We also manually digitized the left and right edges from the 3-D LiDAR data in each road section. The automated extracted 3-D edges are represented in red, while the manually digitized 3-D edges are represented in blue in the first, second, and third road sections in Figs. 8-10, respectively. In the next section, we validate the experimental results using our road edge validation approach and discuss them.

\section{RESULTS AND DISCUSSION}

We applied our validation approach to estimate the accuracy of the automatically extracted 3-D road edges in three tested road sections. Box plots for the accuracy values of these extracted left and right edges in the first, second, and third road sections are shown in Figs. 11-13, respectively. We also carried out statistical analyses of the accuracy values for these extracted edges in the first, second, and third road sections as shown in Table V. Our automated algorithm successfully extracted the left and right edges in all three road sections. In the first road section, accuracy values were found to be better than in the second and third road sections. The minimum-maximum and 

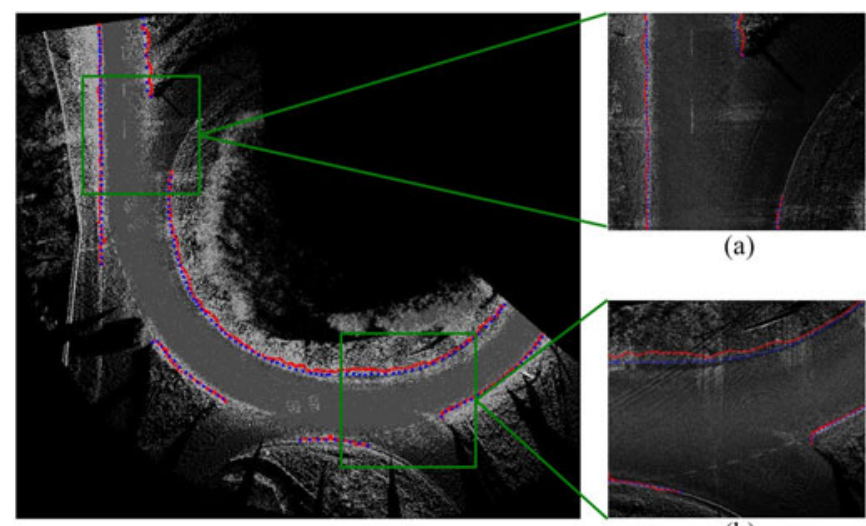

(a)

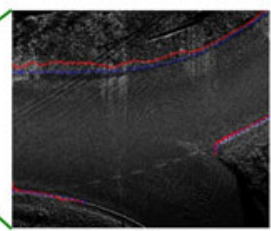

(b)

Fig. 9. Automatically extracted 3-D edges are represented in red and the manually digitized 3-D edges are represented in blue in the second 105-m road section. The inset pictures (a) and (b) of left and right edges show some of the points in detail.
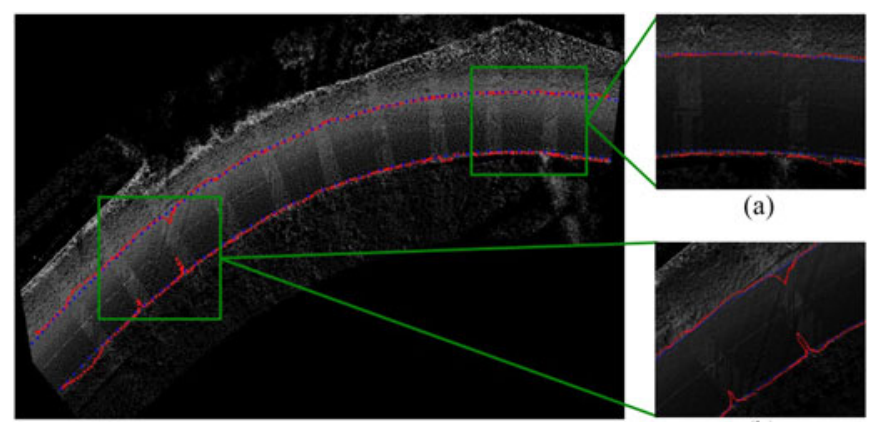

(a)

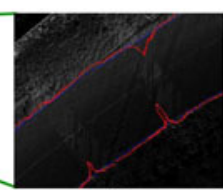

(b)

Fig. 10. Automatically extracted 3-D edges are represented in red and the manually digitized 3-D edges are represented in blue in the third 90-m road section. The inset pictures (a) and (b) of left and right edges show some of the points in detail.

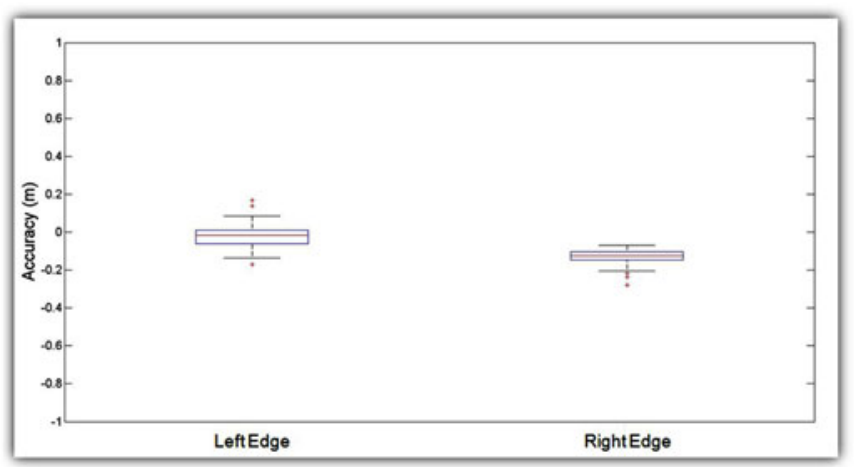

Fig. 11. Box plot for the accuracy values of the automatically extracted left and right edges in the first $50-\mathrm{m}$ road section.

lower-upper adjacent range values were found to be lowest in the first section, while its left and right edge accuracy values were within a $\pm 0.2 \mathrm{~m}$ and $\pm 0.3 \mathrm{~m}$ tolerance, respectively. In the second road section, $3.15 \%$ left edge and $54.33 \%$ right edge accuracy values were more than $\pm 0.3 \mathrm{~m}$ tolerance, while in the third road section, $16.36 \%$ left edge and $53.64 \%$ right edge

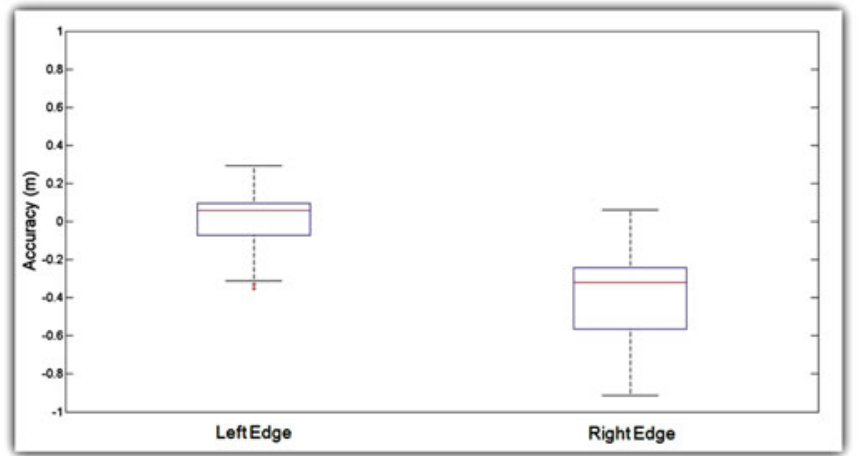

Fig. 12. Box plot for the accuracy values of the automatically extracted left and right edges in the second 105-m road section.

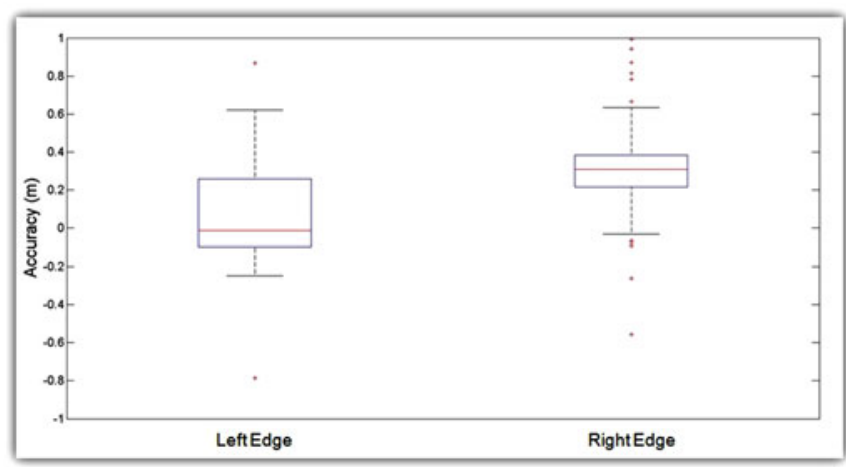

Fig. 13. Box plot for the accuracy values of the automatically extracted left and right edges in the third $90-\mathrm{m}$ road section.

accuracy values were found to be outside the $\pm 0.3 \mathrm{~m}$ tolerance. The higher accuracy level in the first road section was attained due to the uniform and dense point cloud along it, which led to the generation of smooth raster surfaces. The use of such data enabled the snake curve to precisely converge to the road edges.

In the first road section, the left edge displayed slightly better results than the right edge. The minimum-maximum and lowerupper adjacent range values were lower for the right edge; however, the percentages of accuracy inside $\pm 0.01, \pm 0.1$, and \pm 0.2 $\mathrm{m}$ were higher for the left edge. The accuracy level in the first section can be further improved with the use of a reflectance attribute which is a representation of the normalized intensity values. The intensity values are required to be normalized with respect to illuminated surface characteristics, distance from the laser scanner to the illuminated surface, and incidence angle of the laser pulse [34]. This will allow us to assign a height weight to the reflectance GVF energy in the algorithm. The use of a pulse width attribute will also improve the accuracy values in the first road section.

In the second and third road sections, the left edge accuracyvalues were better than the right edge. In the second section, the minimum-maximum and lower-upper adjacent range values were lower for the left edge, while in the third section, these range values were slightly lower for the right edge. In both these sections, the highest percentages of accuracy inside 
TABLE V

STATISTICAL ANALYSIS OF THE ACCURACY VALUES OF THE AUTOMATICALLY EXTRACTED LEFT AND RIGHT EDGES IN THE FIRST, SECOND, AND THIRD ROAD SECTIONS

\begin{tabular}{|c|c|c|c|c|c|c|}
\hline & \multicolumn{3}{|c|}{ Left Edge } & \multicolumn{3}{|c|}{ Right Edge } \\
\hline & $\begin{array}{c}\text { First } \\
\text { Section }\end{array}$ & $\begin{array}{l}\text { Second } \\
\text { Section }\end{array}$ & $\begin{array}{l}\text { Third } \\
\text { Section }\end{array}$ & $\begin{array}{c}\text { First } \\
\text { Section }\end{array}$ & $\begin{array}{l}\text { Second } \\
\text { Section }\end{array}$ & $\begin{array}{l}\text { Third } \\
\text { Section }\end{array}$ \\
\hline minimum (m) & -0.17 & -0.35 & -0.79 & -0.28 & -0.92 & -0.56 \\
\hline $\operatorname{maximum}(\mathrm{m})$ & 0.17 & 0.29 & 0.87 & -0.07 & 0.06 & 0.99 \\
\hline $\begin{array}{l}\text { lower adjacent } \\
\text { (m) }\end{array}$ & -0.13 & -0.31 & -0.25 & -0.20 & -0.92 & -0.03 \\
\hline $\begin{array}{l}\text { upper adjacent } \\
\text { (m) }\end{array}$ & 0.09 & 0.29 & 0.62 & -0.07 & 0.06 & 0.63 \\
\hline $\begin{array}{l}\text { 25th percentile } \\
\text { (m) }\end{array}$ & -0.06 & -0.07 & -0.10 & -0.15 & -0.56 & 0.22 \\
\hline $\begin{array}{l}\text { 75th percentile } \\
\text { (m) }\end{array}$ & 0.11 & 0.10 & 0.26 & -0.10 & -0.24 & 0.38 \\
\hline mean $(\mathrm{m})$ & -0.02 & 0.01 & 0.07 & -0.13 & -0.38 & 0.30 \\
\hline median (m) & -0.02 & 0.06 & -0.01 & -0.13 & -0.32 & 0.31 \\
\hline outliers (\%) & 5.08 & 1.57 & 1.82 & 5.08 & 0 & 10 \\
\hline $\begin{array}{l}\text { inside } \pm 0.01 \\
(\%)\end{array}$ & 20.34 & 3.94 & 1.82 & 0 & 0.79 & 0 \\
\hline $\begin{array}{l}\text { inside } \pm 0.1 \\
(\%)\end{array}$ & 89.83 & 55.91 & 36.36 & 20.34 & 10.24 & 8.18 \\
\hline $\begin{array}{l}\text { inside } \pm 0.2 \\
(\%)\end{array}$ & 100 & 86.61 & 64.55 & 91.53 & 21.26 & 16.36 \\
\hline $\begin{array}{l}\text { inside } \pm 0.3 \\
(\%)\end{array}$ & 100 & 96.85 & 83.64 & 100 & 45.67 & 46.36 \\
\hline $\begin{array}{l}\text { outside } \pm 0.3 \\
(\%)\end{array}$ & 0 & 3.15 & 16.36 & 0 & 54.33 & 53.64 \\
\hline $\begin{array}{l}\text { horizontal } \\
\text { RMSE (m) }\end{array}$ & 0.05 & 0.13 & 0.23 & 0.14 & 0.42 & 0.31 \\
\hline $\begin{array}{l}\text { vertical RMSE } \\
\text { (m) }\end{array}$ & 0.05 & 0.04 & 0.10 & 0.03 & 0.16 & 0.24 \\
\hline
\end{tabular}

$\pm 0.01, \pm 0.1, \pm 0.2$, and $\pm 0.3 \mathrm{~m}$ were for the left edges. This lower accuracy for the right edge was due to lower point density along the right side. The use of a modified balloon energy in the second road section provided an additional inflation energy to the snake curve causing it to move toward the right edge; however, it was not helpful in precisely converging the snake curve to the right edge. In the second section, the snake curves were found to be extended along the road-lanes entering into the roundabout. The nonroad edge points in the extended snake curves can be automatically removed with the batch processing of entering road-lanes. In the third road section, the reflectance values were not accurately normalized, which led to the generation of raster surfaces with road marking cells near the edges. It created an obstruction for the snake curve to move beyond them. The use of a properly normalized reflectance attribute along with a uniform and dense point cloud will provide an improved extraction of road edges in both the sections.

Yang, Fang, and Li [21] and Wang et al. [24] validated the kerb edges extracted from MLS data by detailing an average completeness values of $95.13 \%$ and $95.41 \%$, respectively, while average correctness values of $97.04 \%$ and $99.35 \%$, respectively. Guan, Li, Yu, Chapman, and Wang [23] reported an average horizontal and vertical root mean square error (RMSE) values of 0.08 and $0.02 \mathrm{~m}$, respectively, for the kerb edges extracted from MLS data. In comparison, our validation approach provided a more qualitative evaluation of extracted kerb edges based on estimating their orthogonal proximity from the manually digitized edges. Our automated algorithm extracted the kerb edges along right side of first road section and along both sides of second road section with an average horizontal and vertical RMSE values of 0.23 and $0.08 \mathrm{~m}$, respectively. This accuracy can be further improved with the use of dense point cloud data along both sides of road section and proper utilization of all the LiDAR attributes in our algorithm.

We analyzed the computational performance of our algorithm by estimating the total time taken by the snake curve to move from its initial position to the final position in each tested road section. In the first, second, and third road sections, the snake curve took an approximate 624,1357 , and 864 s, respectively, to converge to the road edges. This analysis was performed on a computer with Intel Core i7-4610M processor @ $3 \mathrm{GHz}, 8-\mathrm{GB}$ RAM, and a 64-bit operating system.

\section{CONCLUSION}

Our algorithm is based on the novel integration of GVF and balloon snake models to extract road edges from MLS data. The algorithm was tested on datasets which were acquired using multiple MLS systems along complex road sections. The successful extraction of road edges from the sections consisting of distinct sets of edges, a roundabout, and a high degree of curvature validates our algorithm. We presented a detailed analysis of the internal and external energy parameters involved in our algorithm. The use of these optimal values enabled automation of the process of extracting road edges for all the tested road sections. We modified the balloon external energy and presented its utility for extracting road edges from the datasets with low and nonuniform point densities. Our novel road edge validation approach negated various limitations associated with traditional approaches, which are based on computing quality measure values with respect to buffer zones created around reference data. The presented approach enables us to efficiently validate the extracted edges in the tested road sections by directly estimating their orthogonal proximity from the reference edges.

The performance of our algorithm improved significantly with dense and uniform point cloud along the road sections. It could be further improved with the use of a reflectance attribute with proper normalized values. This will help distinguish the road surface from the grass-soil and the kerb edges more accurately. Further research is required to investigate the applicability of the geometric active contour models for extracting road edges. This could be advantageous as it will remove the requirement for weighting various input parameters. The urban road sections are often accompanied with stationary vehicles or pedestrians during the data acquisition process, which can lead to missing points in the LiDAR data section. The missing points introduce noisy cells along the raster surface generated from the LiDAR data. This noise can be removed with an optimal use of hierarchical thresholding and modified balloon energy approaches, which are required to be further analyzed. We intend to develop an error correction approach which, when incorporated in our algorithm, will remove false road edges caused by false positives or occlusions. Large-scale implementation of our algorithm will be enabled through the ongoing construction of a 
geospatial data management system to handle 100s of kilometers of LiDAR data.

\section{ACKNOWLEDGMENT}

The authors would like to acknowledge the 3D Laser Mapping company for providing the Streetmapper MLS data.

\section{REFERENCES}

[1] WHO, "Global status report on road safety," 2015. [Online]. Available: http://www.who.int/violence_injury_prevention

[2] G. Gatti, C. Polidori, I. Galvez, K. Mallschutzke, R. Jorna, M. Leur, M. Dietze, D. Ebersbach, C. Lippold, B. Schlag, G. Weller, A. Wyczynski, F. Iman, and C. Aydin, Safety Handbook for Secondary Roads, 2007. [Online]. Available: http://ec.europa.eu/transport/roadsafety_library

[3] UNECE, Transport Review: Road Safety, 2008. [Online]. Available: http://www.unece.org

[4] ERSO, "Roads," 2006. [Online]. Available: http://ec.europa.eu

[5] T. McCarthy and C. P. McElhinney, "European Road Safety Inspection (EuRSI) research project," presented at the Eur. Transport Conf., Glasgow, U.K., Oct. 11-13, 2010.

[6] P. Kumar, C. P. McElhinney, P. Lewis, and T. McCarthy, "An automated algorithm for extracting road edges from terrestrial mobile LiDAR data," ISPRS J. Photogrammetry Remote Sens., vol. 85, pp. 44-55, 2013.

[7] M. Kass, A. Witkin, and D. Terzopoulos, "Snakes: Active contour models," Int. J. Comput. Vision, vol. 1, no. 4, pp. 321-331, 1988.

[8] P. Kumar, "Road features extraction using terrestrial mobile laser scanning system," Ph.D. dissertation, Nat. Univ. Ireland Maynooth, Maynooth, Ireland, 2012, p. 300.

[9] V. Caselles, F. Catt, T. Coll, and F. Dibos, "A geometric model for active contours in image processing," Numerische Math., vol. 66, pp. 1-31, 1993.

[10] R. Malladi, J. A. Sethian, and B. C. Vemuri, "Shape modeling with front propagation: A level set approach," IEEE Trans. Pattern Anal. Mach. Intell., vol. 17, no. 2, pp. 158-175, Feb. 1995.

[11] G. Vosselman and Z. Liang, "Detection of curbstones in airborne laser scanning data," Int. Archives Photogrammetry, Remote Sens. Spatial Inf. Sci., vol. 38, no. Part 3/W8, pp. 111-116, 2009.

[12] C. Heipke, H. Mayer, C. Wiedemann, and O. Jamet, "Evaluation of automatic road extraction," Int. Archives Photogrammetry, Remote Sens. Spatial Inf. Sci., vol. 32, no. Part 2/3W3, pp. 151-160, 1997.

[13] S. J. O. Elberink and G. Vosselman, "Quality analysis of 3D road reconstruction," Int. Archives Photogrammetry, Remote Sens. Spatial Inf. Sci., vol. 36, no. Part 3/W52, pp. 1-6, 2007.

[14] S. O. Elberink and G. Vosselman, "3D information extraction from laser point clouds covering complex road junctions," Photogrammetric Rec., vol. 24, no. 125 , pp. 23-36, 2009.

[15] A. Jaakkola, J. Hyyppä, H. Hyyppä, and A. Kukko, "Retrieval algorithms for road surface modelling using laser based mobile mapping," Sensors, vol. 8, no. 9, pp. 5238-5249, Sep. 2008.

[16] J. Goepfert and F. Rottensteiner, "Adaption of roads to ALS data by means of network snakes," Int. Archives Photogrammetry, Remote Sens. Spatial Inf. Sci., vol. 38, no. 3/W8, pp. 24-29, 2009.

[17] A. Boyko and T. Funkhouser, "Extracting roads from dense point clouds in large scale urban environment," ISPRS J. Photogrammetry Remote Sens., vol. 66, no. 6, pp. S2-S12, 2011.

[18] S. Ibrahim and D. Lichti, "Curb-based street floor extraction from mobile terrestrial LiDAR point cloud," Int. Archives Photogrammetry, Remote Sens. Spatial Inf. Sci., vol. 39, no. Part B5, pp. 193-198, 2012.

[19] L. Zhou and G. Vosselman, "Mapping curbstones in airborne and mobile laser scanning data," Int. J. Appl. Earth Observ. Geoinf., vol. 18, pp. 293-304, 2012.

[20] A. Serna and B. Marcotegui, "Urban accessibility diagnosis from mobile laser scannning data," ISPRS J. Photogrammetry Remote Sens., vol. 84, pp. 23-32, 2013.

[21] B. Yang, L. Fang, and J. Li, "Semi-automated extraction and delineation of 3D roads of street scene from mobile laser scanning point clouds," ISPRS J. Photogrammetry Remote Sens., vol. 79, pp. 80-93, 2013.

[22] H. Guan, J. Li, Y. Yu, C. Wang, M. Chapman, and B. Yang, "Using mobile laser scanning data for automated extraction of road markings," ISPRS J. Photogrammetry Remote Sens., vol. 87, pp. 93-107, 2014.

[23] H. Guan, J. Li, Y. Yu, M. Chapman, and C. Wang, "Automated road information extraction from mobile laser scanning data," IEEE Trans. Intell. Transp. Syst., vol. 16, no. 1, pp. 194-205, Feb. 2015.
[24] H. Wang et al. "Road boundaries detection based on local normal saliency from mobile laser scanning data," IEEE Geosci. Remote Sens. Lett., vol. 12, no. 10, pp. 2085-2089, Oct. 2015.

[25] P. Kumar, P. Lewis, and C. P. McElhinney, "Parametric analysis for automated extraction of road edges from mobile laser scanning data," Int Annal. Photogrammetry, Remote Sens. Spatial Inf. Sci., vol. II-2/W2, pp. 215-221, 2015.

[26] C. Crawford, "Minimizing noise from LiDAR for contouring and slope analysis," 2009. [Online]. Available: http://blogs.esri.com/esri/arcgis/ 2009/09/02

[27] ESRI, "Terrain pyramids," 2010. [Online]. Available: http://webhelp.esri. com/ArcGISdesktop/9.3

[28] C. Xu and J. L. Prince, "Gradient vector flow: A new external force for snakes," in Proc. Comput. Vision Pattern Recog., San Juan, PR, USA, 1997, pp. 66-71.

[29] M. Sonka, V. Hlavac, and R. Boyle, Image Processing, Analysis and Machine Vision 2nd ed. New York, NY, USA: Thomson Eng., 2008.

[30] J. Canny, "A computational approach to edge detection," IEEE Trans. Pattern Anal. Mach. Intell., vol. PAMI-8, no. 6, pp. 679-698, Nov. 1986.

[31] C. Cahalane, C. P. McElhinney, P. Lewis, and T. McCarthy, "Calculation of target-specific point distribution for 2D mobile laser scanners," Sensors, vol. 14, no. 6, pp. 9471-9488, 2014.

[32] StreetMapper, "3D laser mapping," 2015. [Online]. Available: http://www. 3dlasermapping.com/streetmapper

[33] RouteMapper, "IBI Group," 2015. [Online]. Available: http://www. routemapper.net

[34] S. Kaasalainen, A. Jaakkola, M. Kaasalainen, A. Krooks, and A. Kukko, "Analysis of incidence angle and distance effects on terrestrial laser scanner intensity: Search for correction methods," Remote Sens., vol. 3, no. 10 , pp. 2207-2221, 2011.

Pankaj Kumar received the Ph.D. degree from the National Centre for Geocomputation, Maynooth University, Maynooth, Ireland, in 2012.

$\mathrm{He}$ is currently a Senior Lecturer with the Department of Geoinformation, Universiti Teknologi Malaysia, Johor Bahru, Malaysia. His research interests include LiDAR, 2-D and 3-D geospatial data analysis, navigation, numerical modeling, and object segmentation.

Paul Lewis received the Ph.D. degree in computer science and geocomputation from the National Centre for Geocomputation (NCG), Maynooth University, Maynooth, Ireland, in 2009.

Since then, he has been a Research Fellow with the NCG in a research commercialization capacity.

Conor P. McElhinney, photograph and biography not available at the time of publication.

Pawel Boguslawski received the Ph.D. degree from the University of South Wales, Newport, UK, in 2011.

He is currently a Researcher with the Faculty of Environment and Technology, University of the West of England, Bristol, UK. His research interests include 3D spatial modeling, data structures, indoor navigation, and emergency response.

Dr. Boguslawski is the Co-Chairman of the ISPRS Working Group II/2 Multiscale $N$-dimensional Spatial Data Representations, Data Structures and Algorithms and ISPRS UV Helava Award jury member.

Tim McCarthy received the Ph.D. degree from the University of London (Birkbeck), London, U.K., in 1999.

$\mathrm{He}$ is currently a Principal Investigator with the National Centre for Geocomputation, Maynooth University, Maynooth, Ireland. He has more than 25 years' experience in researching and developing innovative geospatial technologies. He is the Irish National Chair for EU Copernicus Program and is actively engaged in geospatial research across various sectors and currently providing technology support to a number of start-ups ventures based around mobile devices and drones. 